\title{
Engineering Metal Adhesion Layers That Do Not Deteriorate Plasmon Resonances
}

\author{
Thomas Siegfried, ${ }^{\dagger, *}{ }^{\text {Yasin Ekinci, }}{ }^{\dagger}$ Olivier J.F. Martin, ${ }^{\ddagger}$ and Hans Sigg ${ }^{\dagger}$ \\ ${ }^{\dagger}$ Laboratory for Micro- and Nanotechnology, Paul Scherrer Institute, 5232 Villigen-PSI, Switzerland and ${ }^{\ddagger}$ Nanophotonics and Metrology Laboratory, EPFL, \\ 1015 Lausanne, Switzerland
}

\begin{abstract}
Adhesion layers, required to stabilize metallic nanostructures, dramatically deteriorate the performances of plasmonic sensors, by severely damping the plasmon modes. In this article, we show that these detrimental effects critically depend on the overlap of the electromagnetic near-field of the resonant plasmon mode with the adhesion layer and can be minimized by careful engineering of the latter. We study the dependence of the geometrical parameters such as layer thickness and
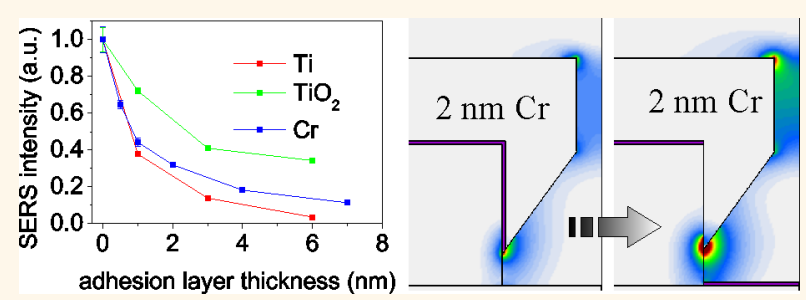

shape on the near-field of localized plasmon resonances for traditional adhesion layers such as $\mathrm{Cr}$, $\mathrm{Ti}$, and $\mathrm{TiO}_{2}$. Our experiments and simulations reveal a strong dependence of the damping on the layer thickness, in agreement with the exponential decay of the plasmon near-field. We developed a method to minimize the damping by selective deposition of thin adhesion layers $(<1 \mathrm{~nm})$ in a manner that prevents the layer to overlap with the hotspots of the plasmonic structure. Such a designed structure enables the use of standard $\mathrm{Cr}$ and Ti adhesion materials to fabricate robust plasmonic sensors without deteriorating their sensitivity.
\end{abstract}

KEYWORDS: adhesion layer · damping · surface-enhanced Raman scattering · near-field simulation · localized plasmon resonance · biosensors

( reat amounts of numerical and experimental investigations have been devoted to boosting the signal of plasmonic sensors fabricated from noble metal nanoantennas with sharp edges or with gaps separated by a few nanometers. ${ }^{1-8}$ The field enhancement depends mainly on the ability to couple incident photons to localized surface plasmon modes. Such resonant modes are prone to damping by scattering and absorption in the metal and its surrounding materials, thus, limiting the achievable enhancement. ${ }^{9}$ By interference with subradiant modes, as in Fano resonant systems, radiative losses can be reduced, but not suppressed completely. ${ }^{10,11}$ In any case, the field enhancement is often severely reduced by the presence of adhesion layers. These layers are required to pin the noble metals to the substrate, particularly when the fabrication requires lift-off and sonication or when the applications demand high structural robustness. ${ }^{12}$ Thanks to their broad availability and process compatibility with the evaporation of the noble metals, $\mathrm{Cr}$ and $\mathrm{Ti}$ are the most commonly used adhesion materials, with typical thicknesses ranging between 1 and $10 \mathrm{~nm} .^{13-15}$ Although the existence of the adhesion layer is an important part of the plasmonic structure, its influence on the near and far field of the plasmonic modes is often neglected., 90 The perturbation of the plasmon resonance can be derived from the real and imaginary parts of the adhesion materials dielectric function which affects the refractive index locally and introduces absorption. Under such circumstances, surface and localized surface plasmon resonances (SPR and LSPR) are red-shifted and broadened, leading to reduced signal sensitivities, caused by the reduced quality factor $\mathrm{Q}$ of the resonant mode. ${ }^{17-21}$ As a consequence, processes where high near-field amplitudes are needed, such as surface enhanced Raman scattering (SERS) and fluorescence, ${ }^{16,21-23}$ as well as particle trapping, ${ }^{24}$ are weakened.

A route to reduce damping is the use of less absorptive adhesion materials such as $\mathrm{Cr}_{2} \mathrm{O}_{3}, \mathrm{TiO}_{2}{ }^{16,25}$ and $\mathrm{ITO}^{26}$ although these
* Address correspondence to thomas.siegfried@psi.ch.

Received for review January 14, 2013 and accepted February 22, 2013.

Published online February 22, 2013 $10.1021 / \mathrm{nn} 4002006$

Cㄱ 2013 American Chemical Society 
oxides require more specialized preparation and equipment. Another alternative is the use of a selfassembled monolayer such as mercaptosilane, where the silane binds to oxidic surfaces and the thiol end group binds to noble metals. ${ }^{27}$ The dielectric environment of the noble metal is found almost unaffected by such a monolayer, and thus, the induced plasmon resonance shift and near-field damping are minimal. ${ }^{9,21}$ Unfortunately, the utilization of molecular adhesion layers has severe drawbacks as it requires additional fabrication steps either in wet $^{21}$ or dry ${ }^{9}$ conditions and the layers may not withstand oxygen plasma or ultraviolet ozone (UVO) cleaning steps indispensible for reusable plasmonic sensors. ${ }^{12}$

In this work, we systematically investigate the damping effect of adhesion layers on the near field enhancement of $\mathrm{Au}$ nanostructures. We provide a nanometer precise study of the thickness dependency of metal and metal oxide adhesion layers on the nearand far-field properties of plasmonic nanostructures using SERS and reflection spectroscopy. Additionally, we propose and demonstrate a method to minimize plasmon damping of adhesion layers by excluding the adhesion material from the hotspot (region with the highest near-field enhancement). We further verify for a broad range of Au grating gap sizes and adhesion layer thicknesses that damping is mostly dependent on the overlap between the near-field hotspot and the adhesion layer. Comparison with simulations provides deeper insight into the damping mechanism of adhesion layers and demonstrates how thin adhesion layers can be made without deteriorating their bonding properties. These different strategies enable the realization of plasmonic nanostructures that include metal adhesion layers and do not suffer from damping.

\section{RESULTS AND DISCUSSION}

To study the influence of adhesion layers on the performance of plasmonic structures, we utilize periodic nanogap arrays. These were fabricated in a single-step lithography and angular evaporation process. Onedimensional gratings of photoresists with various thicknesses and duty cycles were obtained reproducibly over large areas and with high throughput using extreme ultraviolet interference lithography (EUV-IL). ${ }^{28}$ Hydrogen silsesquioxane (HSQ) was used as photoresist, which is converted into $\mathrm{SiO}_{2}$ upon exposure and development. The adhesion layer and noble metal were evaporated successively onto the photoresist under glancing angles. The substrate was repeatedly tilted to the opposite angle, as shown in Figure $1 \mathrm{a}$, to homogeneously cover the photoresist and controllably reduce the size of the gap. ${ }^{29}$ With this technique, we fabricated nanogap patterns with variable thicknesses and gap sizes, with the cross section shown in Figure $1 \mathrm{~b}$.

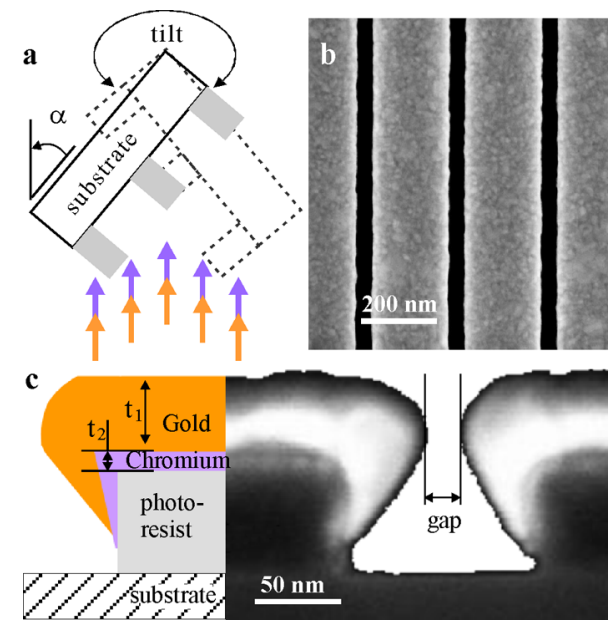

Figure 1. (a) Schematic description of the angular evaporation method, resulting in periodic nanogap arrays. (b) Top view scanning electron micrograph (SEM) and (c) schematic view and SEM of the cross section of the nanogap arrays with the adhesion and gold layers. The pattern is characterized by the gold thickness $t_{1}$, the adhesion layer thickness $t_{2}$ and the pattern gap size. The cross section is reminiscent of a crescent.

The thus obtained nanostructures consist of an array of crescent shaped line gratings. This configuration, as we will show later, facilitates the generation of strong electromagnetic fields near the crescent tips when the incident light field couples resonantly to the gap-mode of the periodic array. ${ }^{10}$ Our nanometer precise and widely adjustable evaporation technique allowed us to study the damping effect of several adhesion layer parameters. The near-field amplitude was quantified using the SERS signal intensity. Thanks to our gentle fabrication process, adhesion layer free patterning was also possible, which we attribute to the clasping of the evaporated gold around the photoresist grating.

The far- and near-field response of our nanogap pattern are simulated for adhesion layers of various thicknesses using a 3D finite element method based on the surface integral solution of Maxwell's equations. ${ }^{30,31}$ The far-field spectra in reflection for increasing $\mathrm{Cr}$ adhesion layer thicknesses are shown in Figure 2 a. The distinctive dip at $680 \mathrm{~nm}$ wavelength is attributed to the resonant excitation of the plasmon gap mode, similar to enhanced optical transmission (EOT). ${ }^{32,33}$ This resonance exhibits a red-shift with increasing $\mathrm{Cr}$ layer thickness, while its amplitude strongly decreases. The second reflection minimum below $500 \mathrm{~nm}$ is attributed to the absorbing interband transitions of gold.

At the resonance wavelength of $680 \mathrm{~nm}$, localized plasmons produce a strong near-field at the metal tips of the crescent pattern, as is visible in Figure $2 \mathrm{~b}$. The amplitude of this hotspot near the sharp crescent tips significantly decreases for increasing adhesion layer thickness, whereas the near-field in the upper gap region seems not at all affected by the adhesion layer. This suggests that the amplitude of the near-field 

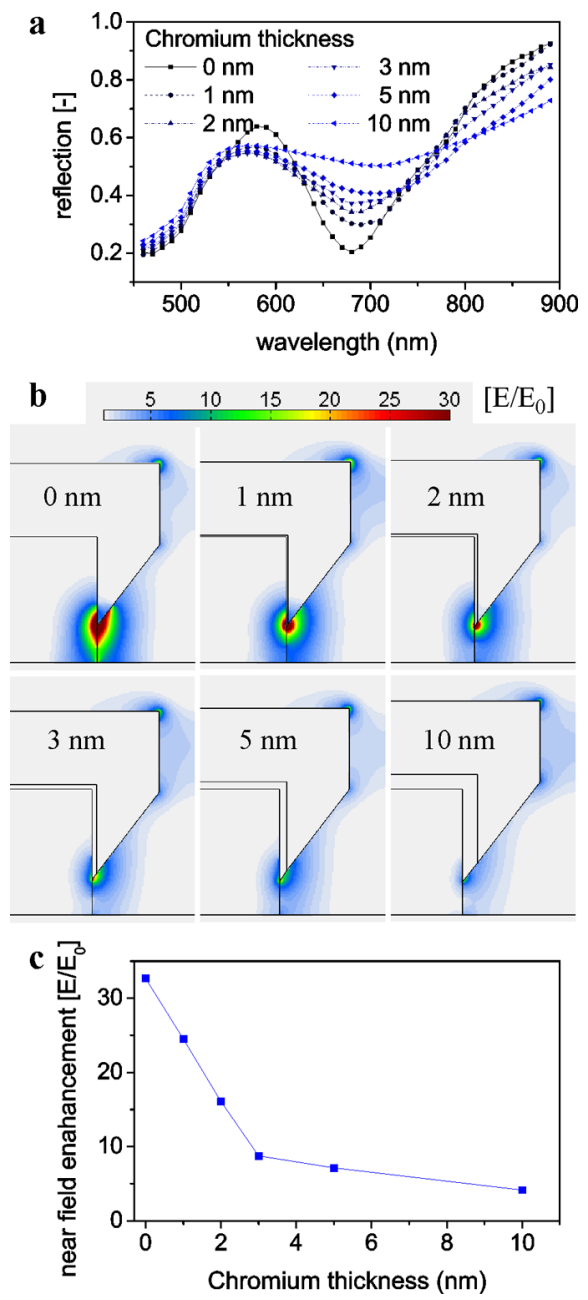

Figure 2. Simulations of the far- and near-field for increasing adhesion layer thicknesses of the crescent pattern with $50 \mathrm{~nm}$ Au gap and a period of $250 \mathrm{~nm}$. (a) Far-field reflection spectra, (b) near-field amplitude maps of the crescent array calculated at a wavelength of $680 \mathrm{~nm}$ and (c) near-field enhancement, $E / E_{0}$, within $5 \mathrm{~nm}$ of the crescent tips. The complex refractive indices $(n, k)$ at a wavelength of $680 \mathrm{~nm}$ for the $80 \mathrm{~nm}$ high photoresist pattern, the $\mathrm{Cr}$ with thickness between 0 and $10 \mathrm{~nm}$ and the $50 \mathrm{~nm}$ Au layer were taken to be $(1.39,0),(3.07,3.36)$, and $(0.135,3.88)$, respectively. The substrate was silicon $(3.81,0.0024)$. The polarization of the electric field is set across the gaps.

is locally weakened, while the plasmonic mode of the crescent pattern is nearly not altered. We will show that the origin of this phenomenon is the close contact between the electrical field at the hotspot and the adhesion layer. The dependence on the adhesion layer thickness of the damping of the electrical field at the hotspot is shown in Figure 2c. The damping increases strongly with the adhesion layer thickness, resulting in a dramatic loss of the near-field enhancement by a factor of 5 already for a $3 \mathrm{~nm}$ thick adhesion layer. This near-field damping on the layer thickness is congruent to what we observe in the SERS measurements, shown below.

The corresponding experiments were performed for adhesion layers made of $\mathrm{Cr}, \mathrm{Ti}$ and $\mathrm{TiO}_{2}$. The $\mathrm{Au}$ was
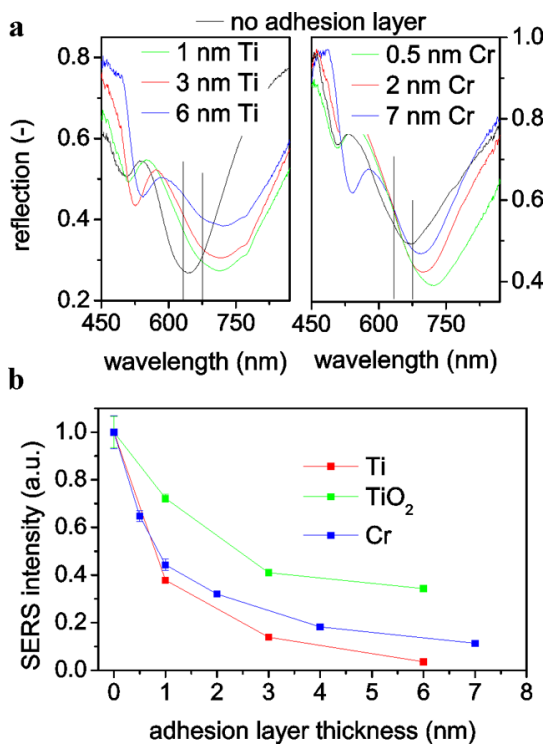

Figure 3. Dependence of the adhesion layer thickness on (a) the reflection spectra for $\mathrm{Ti}$ and $\mathrm{Cr}$ coatings and (b) the SERS intensity for $\mathrm{Ti}_{1} \mathrm{TiO}_{2}$ and $\mathrm{Cr}$ coatings. The laser excitation wavelength at $633 \mathrm{~nm}$ and the recorded SERS wavelength at $676 \mathrm{~nm}$, corresponding to a Raman shift of $1008 \mathrm{~cm}^{-1}$, are marked in the reflection spectra. The SERS signal is recorded from a self-assembled benzene-ethane-thiol monolayer deposited on a line array of $50 \mathrm{~nm}$ thick Au approximately $40 \mathrm{~nm}$ wide gaps. The polarization of the electric field is set across the gaps. Error bars, often smaller than the icon size, represent the standard deviation for 16 spatially separated SERS measurements.

always evaporated in parallel for each sample series of varying thicknesses, to minimize the influence of metal roughness deviations. Reflection spectra are shown for $\mathrm{Ti}$ and $\mathrm{Cr}$ in Figure $3 \mathrm{a}$ and indicate $\mathrm{a} 70 \mathrm{~nm}$ resonance red-shift, independent of the Ti layer thickness, and a roughly $30 \mathrm{~nm}$ red-shift for $\mathrm{Cr}$, slightly changing with the thickness. The resonance broadens and its amplitude decreases with increasing $\mathrm{Ti}$ and $\mathrm{Cr}$ layer thicknesses, in agreement with the simulation on $\mathrm{Cr}$ shown in Figure 2a. The observed blue-shift for increasing the $\mathrm{Cr}$ adhesion layer thickness is attributed to an Au gap size reduction of about $5 \mathrm{~nm}$ observed when the adhesion layer thickness is increased from 0 to $7 \mathrm{~nm}$. The deviation between $\mathrm{Ti}$ and $\mathrm{Cr}$ with no adhesion layer could be attributed to a partial liftoff of the Au grating in the $\mathrm{Cr}$ sample, caused by weak bonding.

Figure $3 \mathrm{~b}$ shows the SERS signal intensity dependence on the layer thickness for the three investigated adhesion materials: $\mathrm{Ti}_{1} \mathrm{TiO}_{2}$, and $\mathrm{Cr}$. It is also compared to the near-field decay averaged around the full $\mathrm{Au}$ surface. The samples were prepared with a monolayer of benzene-ethane-thiol and were excited and measured at a wavelength of 633 and $676 \mathrm{~nm}$, respectively, corresponding to a Raman shift of $1008 \mathrm{~cm}^{-1}$. We observe indeed a strong reduction of the SERS intensity for adhesion layer thicknesses up to $3 \mathrm{~nm}$. For thicker layers, this damping of SERS levels off, 
especially for the $\mathrm{Cr}$ adhesion coating. To quantify the strength of the SERS damping, the evolution of the normalized intensity $I / I_{0}$ on the adhesion layer thickness is fitted with a power law of the form $(1+x / n m)^{-a}$, where $x$ is the adhesion layer thickness in nanometers and the exponent $a$ describes the damping rate. An exponent of roughly 0.5 was found for $\mathrm{TiO}_{2}, 1$ for $\mathrm{Cr}$ and roughly 1.5 for Ti. Details of the fitting procedure are given in the Supporting Information, Figure S3. For an adhesion layer thickness of about $6 \mathrm{~nm}$, the SERS intensity was damped by a factor of 2.5 for $\mathrm{TiO}_{2}$, by 10 for $\mathrm{Cr}$ and by 25 for $\mathrm{Ti}$.

As expected, the damping is the smallest for $\mathrm{TiO}_{2}$, since this material has negligible absorption at this wavelength. The residual damping of $a=0.5$ is in part attributed to an incomplete Ti oxidation during evaporation under partial oxygen pressure. The SERS damping is considerably stronger for the metals, because of their large absorption coefficients related to the complex refractive index, $n+i k$. The SERS signal can also decay because of a shift of the resonance, in which case the plasmonic mode is less effectively coupled to the external excitation. ${ }^{22,34}$ For $\mathrm{Ti}$ and $\mathrm{TiO}_{2}$, the induced red-shift was around $70 \mathrm{~nm}$, while for $\mathrm{Cr}$, we have observed a red-shift of only $30 \mathrm{~nm}$, shown in Figure 3a and additionally in Figure $\mathrm{S} 1$ of the Supporting Information. This may explain the stronger damping of Ti despite its smaller absorption coefficient. In fact, as shown in Figure 3a, the overlap of the excitation and Raman scattering wavelength with the resonance wavelength is best for the sample without an adhesion layer and better for $\mathrm{Cr}$ adhesion layers compared to Ti.

The sharp decrease of SERS intensity for adhesion layer thicknesses up to about $3 \mathrm{~nm}$ is attributed to the spatial extension of the confined plasmons. The nearfield generated by plasmons decays exponentially with the distance from the metal surface and typical decay lengths in metals are in the order of up to $10 \mathrm{~nm} .^{35}$ Hence, when the adhesion material is located within this plasmon decay length, the damping is strongest. The reduced slope of SERS damping for increasing adhesion layer thicknesses mimics the exponential decay of the plasmon near-field. Conversely, when the adhesion layer remains away from any hotspot, the damping should be minimized, as will be demonstrated below.

Modeling the expected SERS damping with a power scaling of $\left|E / E_{0}\right|^{4}$, the slope of the experimentally observed SERS damping is found to be smaller compared to the computed decay of the near-field enhancement from Figure $2 \mathrm{c}$. We attribute this behavior to the idealized geometry used in the computation with a point-shaped hotspot, while the real geometry is prone to roughness and corner rounding that spatially expands the hotspot region. In fact, we will demonstrate in the next paragraph that damping is avoided
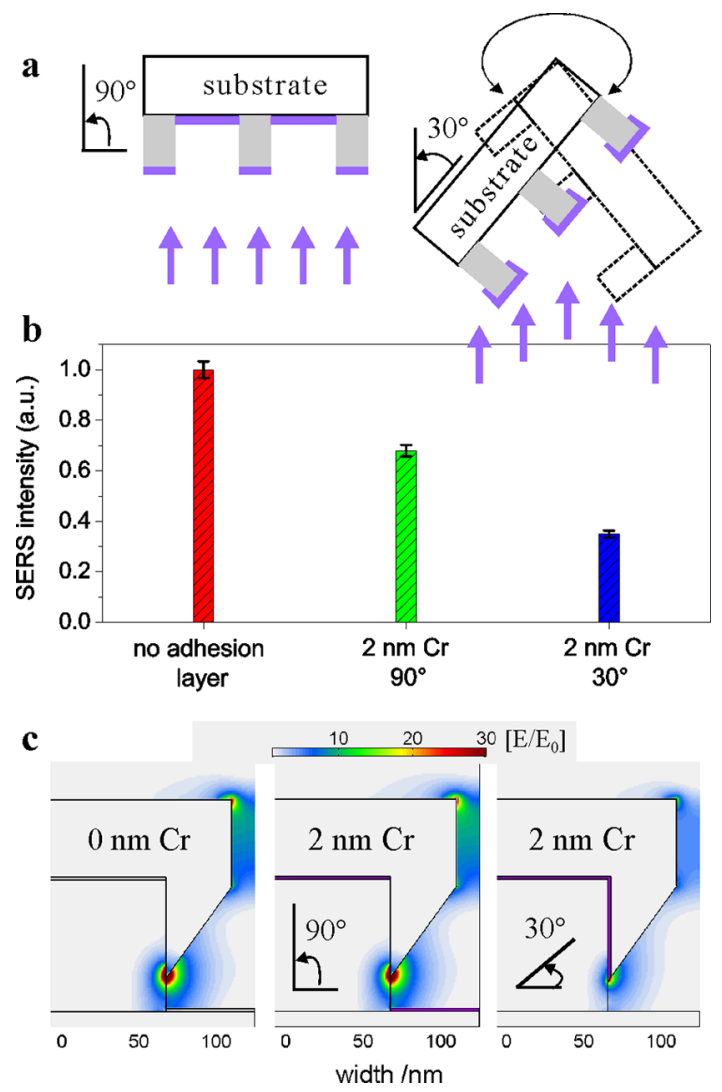

Figure 4. Comparison of SERS signals for vertically and sideward directed $\mathrm{Cr}$ layer deposition. (a) Sample orientation for deposition. (b) Observed SERS damping and (c) simulated near-field amplitude maps for a gold crescent array with a $0 \mathrm{~nm} \mathrm{Cr}$ and $2 \mathrm{~nm} \mathrm{Cr}$ adhesion layers (drawn in purple) evaporated under $90^{\circ}$ and $30^{\circ}$ incidence, calculated at a wavelength of $680 \mathrm{~nm}$. The normalized SERS intensities correspond to the $1008 \mathrm{~cm}^{-1}$ peak obtained from a selfassembled benzene-ethane-thiol monolayer. The polarization of the electric field as in all other experiments is set across the gaps. Error bars represent the standard deviation for 16 spatially separated SERS measurements. The gold thickness for the periodic pattern was $50 \mathrm{~nm}$ and the gap size $30 \mathrm{~nm}$.

when the hotspot is not in direct contact with the adhesion layer.

By changing the evaporation angle for the adhesion material, we could control the contact areas between the adhesion and the Au layers and thus were able to correlate contact area with damping. In the previously discussed experiments, the adhesion material was evaporated at the same angle of $30^{\circ}$ at which the shadow evaporation of Au was performed. This enabled the adhesion layer to fully cover the photoresist grating, including the top and the sidewalls. Evaporating the adhesion layer under normal incidence, however, prevents material deposition on the sidewalls of the resist. In a series of experiments with $2 \mathrm{~nm}$ thick $\mathrm{Cr}$ layers evaporated under $30^{\circ}$ and $90^{\circ}$, we observed that the damping of SERS intensity can indeed be strongly reduced by a factor of 2, as shown in Figure 4a.

Since the plasmonic hotspot is located at the crescent tips, as seen in Figure 2b, we attribute the strong 
a
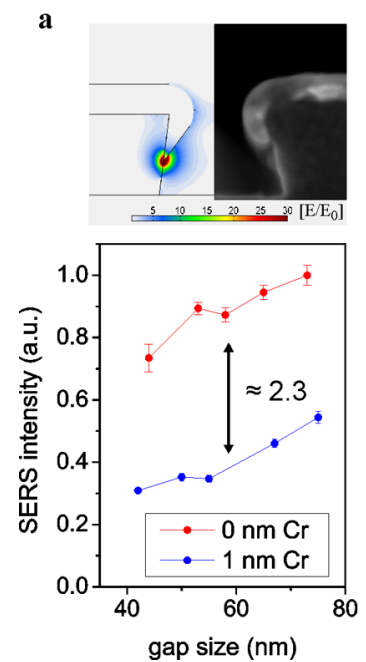

b
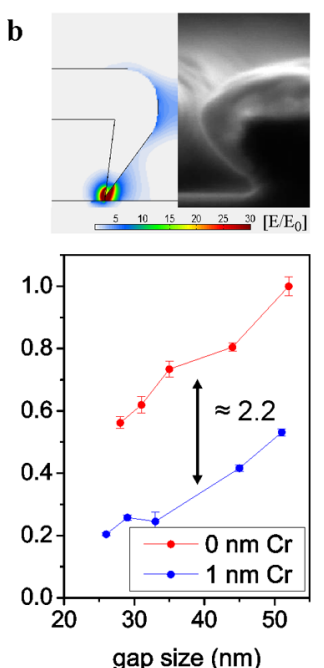

c
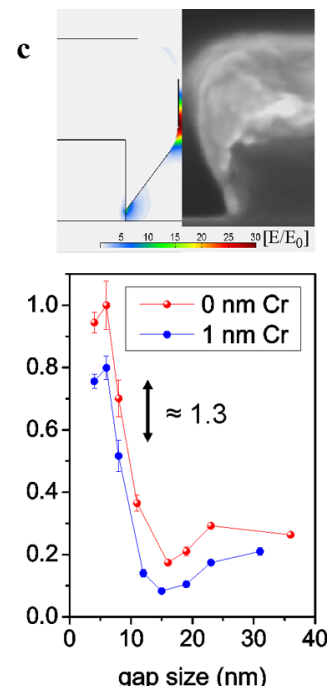

Figure 5. SERS damping for varying gap sizes and metal layer thicknesses of (a) $30 \mathrm{~nm}$, (b) $50 \mathrm{~nm}$, and (c) $80 \mathrm{~nm}$. The $\mathrm{Cr}$ was evaporated under $\pm 30^{\circ}$ from the surface normal. Near-field amplitude maps are compared with cross section scanning electron micrograph images to relate fabrication parameters to the location of hotspots. The polarization of the electric field is set across the gaps. The normalized SERS intensities correspond to the $1008 \mathrm{~cm}^{-1}$ peak of a self-assembled benzeneethane-thiol monolayer. Error bars represent the standard deviation for 16 individual SERS measurements taken at different locations on the substrate.

SERS improvement to the exclusion of the adhesion material from that tip. Simulations performed in conjunction to the experiments show the strongly reduced field $\left(E / E_{0}\right)$ when the adhesion layer is located at the sidewall of the photoresist (Figure 4c, right panel) compared to the case without contact (Figure 4c, center panel). The near-fields and the plasmon resonances (not shown) for the no- and the distant adhesion layer cases are, however, very similar. Compared to the no-adhesion layer case, the average near-field enhancement (averaged over $5 \mathrm{~nm}$ around the hot spot) for the sidewall adhesion layer is about a factor of 3 . The SERS damping is less than we would expect from the simple $\left|E / E_{0}\right|^{4}$ scaling, which we again attribute to the idealized simulation with no roughness and corner rounding. The observed SERS damping, when the adhesion layer is evaporated under $90^{\circ}$, is attributed to an imperfect $90^{\circ}$ alignment of the sample during evaporation and the deposition of $\mathrm{Cr}$ onto the $\mathrm{Si}$ substrate, where it may come into contact with the hotspot, as can be seen from Figure $5 b$.

The simulations and experiments show that an appropriate adhesion layer design derived from the knowledge of the near-field distribution enables minimizing damping. The finding can also be extended to other nanopatterns requiring lift-off where additional underetching into the adhesion layer and the substrate can reduce the overlap of the adhesion layer with the near-field hot spot, thereby lowering damping. ${ }^{36}$

In our experiments, the durability of the pattern is conserved for both evaporation angles, as long as the adhesion layer remains in contact with portions of the $\mathrm{Au}$ interface. This has been verified by treating the different substrates in an ultrasonic bath for several minutes without observing any changes in the SERS intensity. Without an adhesion layer, as shown in Figure $3 b$, the SERS standard deviation within the tested pattern was observed to be larger than $7 \%$, while it was well below $3 \%$ for all the other samples. Intense washing of the substrate and sonication induced a partial lift-off of the Au layer from the photoresist grating when no adhesion layer was used. Remarkably, the pattern did not lift-off for an adhesion layer thickness as thin as $0.5 \mathrm{~nm}$, which was proven by consistent SERS signals for sonication times exceeding $20 \mathrm{~min}$. The same pattern could also withstand several successive cleaning runs without loosing the SERS enhancement. ${ }^{12}$ For those experiments, the analyte was removed by $20 \mathrm{~min}$ exposure to an Ultraviolet source followed by redeposition of the analyte monolayer. These additional experiments are shown in Figure S2 of the Supporting Information.

We have validated our findings for a broader range of pattern gap sizes and metal Au thicknesses: in all cases, the overlap between the adhesion layer and the plasmons electromagnetic field is found to dominate the damping factor. We have analyzed the damping of SERS intensities for a variety of pattern gap sizes and gold thicknesses. In Figure 5, we compare the SERS signal damping between zero and $1 \mathrm{~nm} \mathrm{Cr}$ layer for $\mathrm{Au}$ thicknesses of 30,50 , and $80 \mathrm{~nm}$ and for gap sizes between sub-10 and $75 \mathrm{~nm}$. The gap size of the pattern was set by changing the photoresist duty cycle.

The SERS damping was found to be independent of the nanogap size but depends on the thickness of the $\mathrm{Au}$ layer. For Au thicknesses between 30 and $50 \mathrm{~nm}$, the damping is roughly 2.2 , while it is 1.3 for $80 \mathrm{~nm}$. This behavior is congruent with simulations showing that 
the position of the hotspot does not depend on the gap size but changes its locations from the tip to the nanogap region when the Au thickness becomes larger than about $70 \mathrm{~nm}$, cf. comparison between Figure $5 \mathrm{a}$ and $5 b$ to $5 c$. The damping rate of 1.3 is smaller due to the reduced overlap of adhesion layer and hotspot. For the $80 \mathrm{~nm}$ thick Au layers and gap sizes below $15 \mathrm{~nm}$, the SERS intensity increases strongly due to stronger coupling between the crescents forming a nanogap channel. ${ }^{29}$

\section{CONCLUSION}

We have systematically investigated the geometric constraints associated with adhesion layers such as thickness and overlap with the hotspots and found a route to minimize the near-field damping of localized plasmon resonances. The near-field damping has been quantified by simulations and SERS experiments. The damping has been attributed to the absorption in the adhesion layer, leading to the reduction of the field amplitude, broadening and red shifting up to $70 \mathrm{~nm}$ of the resonance wavelength. We find that adhesion layers with a thickness well below $1 \mathrm{~nm}$ maintain their adhesive properties, while significantly reducing the damping of the SERS intensity. Outstandingly, the exclusion of the adhesion layer from the proximity of the near-field hotspot reduces damping to a level that was previously only obtained by using metal oxides or monolayers of self-assembled mercaptosilanes. Hence, near-field simulations to locate the hotspots precisely and correspondingly adapted evaporation schemes or underetching of metal adhesion layers into the substrate are the key to realize plasmonic nanostructures with negligible impact on the plasmon resonances. This finding can also be extended to other nanopatterns requiring lift-off, where additional underetching into the adhesion layer and substrate can reduce the overlap of the adhesion layer with the near-field hot spot thereby lowering damping. ${ }^{36}$

To conclude, we could demonstrate that traditional metal adhesion layers with a thickness below $1 \mathrm{~nm}$ and deposited with minimal contact to the near-field hotspots produce robust plasmonic nanostructures without deteriorating their performance.

\section{METHODS}

Fabrication of Nanogap Arrays. Extreme ultraviolet interference lithography at the Swiss Light Source ${ }^{28}$ was used to create line patterns with a period of $250 \mathrm{~nm}$ and a height of $80 \mathrm{~nm}$ over an area of $1 \mathrm{~mm}^{2}$ using hydrogen silsesquioxane (HSQ) resist. Glancing angular deposition ${ }^{37}$ was used to thermally evaporate the adhesion material and subsequently Au (99.99\% purity, purchased from Balzers) layer directly onto the photoresist at a base pressure of $2 \times 10^{-6} \mathrm{mbar}$. The substrate was aligned at an angle of $30^{\circ}$ from the surface plane with an azimuthal orientation of the line pattern perpendicular to the gap expansion (Figure 1a). During evaporation, the substrate was tilted to the mirrored direction $\left(-30^{\circ}\right)$ after every deposited $2 \mathrm{~nm}$, until the final thickness was reached. The adhesion materials $\mathrm{Cr}$ and $\mathrm{Ti}$ (both $99.99 \%$ purity, purchased from Sigma Aldrich) were evaporated at the same angle $\left(30^{\circ} \%-30^{\circ}\right)$ as is the Au or, when specified, evaporation has taken place under normal incidence. $\mathrm{TiO}_{2}$ was evaporated using the $\mathrm{Ti}$ source at a partial oxygen pressure of $5 \mathrm{mbar}$. The Au was evaporated in parallel for the investigation of sample sequences with varying adhesion layer parameter, to help the accuracy of the evaluation and minimize the influence of metal roughness deviations. The gap size was set by the duty cycle of the photoresist pattern while keeping the evaporated thickness constant. Further details on the fabrication process of the sub-20 $\mathrm{nm}$ gap pattern have been reported elsewhere. ${ }^{29}$ The adhesion layers were deposited accurately ( $\mathrm{nm}$ precision of thickness) by placing a quartz balance sensor close to the evaporation source. Thereby, the monitored thickness had to be scaled by $1 / 3$ to factor in the $t \sim x^{2}$ dependency of the layer thickness $t$ compared to the distance $x$ from the evaporation source.

Optical Measurements. Reflection spectra were recorded with a spatial resolution of about $10 \mu \mathrm{m}$ using a commercial spectrometer (Sentech FTP) flanged to a microscope (Leica). The incident light was polarized such that the electric field was aligned across the nanogaps. The reflection spectrum obtained from the continuous Au layer on the same substrate served as reference.

The surface-enhanced Raman scattering experiments were performed on a Horiba LabRam HR with a grating resolution of 600 lines $\mathrm{mm}^{-1}$. The excitation source (HeNe laser, $633 \mathrm{~nm}$ ) with an incident power of $2 \mathrm{~mW}$ was focused $(50 \times$, numerical aperture NA 0.5 ) and laterally deflected within an area of $10 \times$ $10 \mu \mathrm{m}^{2}$ of the nanogap pattern to suppress photobleaching. The incident light was linearly polarized with the electric field aligned across the gaps. The average of 16 single spectra was taken over a patterned area of $300 \times 300 \mu \mathrm{m}^{2}$ in order to allow for statistical evaluation. The SERS intensities depicted in all figures correspond to the Raman peak of the $1008 \mathrm{~cm}^{-1}$ vibrational mode from a self-assembled benzene-ethane-thiol monolayer obtained by $12 \mathrm{~h}$ immersion in a $1 \mathrm{mM}$ solution.

Numerical Simulation. The far-field spectra and near-field maps were calculated with a full-field numerical method based on the solution of the surface integral. ${ }^{30}$ The simulation of the crescent grating array was carried out in a 3D unit cell with periodic boundary conditions along and across the gap plane. The polarization of the electric field was set across the gap axis. The permittivities for the Si substrate and $\mathrm{Cr}$ were approximated with bulk values taken from SOPRA; ${ }^{38}$ it is however noted that bulk values can become unreliable for nanometer layer thicknesses. The refractive index of the photoresist HSQ was assumed to be $1.39 ;^{39}$ the permittivity of Au was taken from Johnson and Christy, ${ }^{40}$ and the surrounding medium was air.

Conflict of Interest: The authors declare no competing financial interest.

Acknowledgment. We thank the Swiss National Science Foundation (SNF) for financial support. Part of this work was performed at the Swiss Light Source (SLS), Paul Scherrer Institute, Switzerland.

Supporting Information Available: $\mathrm{Cr}$ and $\mathrm{TiO}_{2}$ reflection spectra; the pattern SERS stability against sonication and UV cleaning; details of the SERS fitting procedure. This material is available free of charge via the Internet at http://pubs.acs.org.

\section{REFERENCES AND NOTES}

1. Cialla, D.; März, A.; Böhme, R.; Theil, F.; Weber, K.; Schmitt, M.; Popp, J. Surface-Enhanced Raman Spectroscopy (SERS): Progress and Trends. Anal. Bioanal. Chem. 2012, 403, 27-54.

2. Biagioni, P.; Huang, J.-S.; Hecht, B. Nanoantennas for Visible and Infrared Radiation. Rep. Prog. Phys. 2012, 75, 024402 . 
3. Zuloaga, J.; Prodan, E.; Nordlander, P. Quantum Description of the Plasmon Resonances of a Nanoparticle Dimer. Nano Lett. 2009, 9, 887-891.

4. Duan, H.; Hu, H.; Kumar, K.; Shen, Z.; Yang, J. K. W. Direct and Reliable Patterning of Plasmonic Nanostructures with Sub-10-nm Gaps. ACS Nano 2011, 5, 7593-7600.

5. Feichtner, T.; Selig, O.; Kiunke, M.; Hecht, B. Evolutionary Optimization of Optical Antennas. Phys. Rev. Lett. 2012, 109, 127701.

6. Lindquist, N. C.; Nagpal, P.; McPeak, K. M.; Norris, D. J.; Oh, S.-H. Engineering Metallic Nanostructures for Plasmonics and Nanophotonics. Rep. Prog. Phys. 2012, 75, 036501.

7. Lindquist, N. C.; Nagpal, P.; Lesuffleur, A.; Norris, D. J.; Oh, S.-H. Three-Dimensional Plasmonic Nanofocusing. Nano Lett. 2010, 10, 1369-1373.

8. Nagpal, P.; Lindquist, N. C.; Oh, S.-H.; Norris, D. J. Ultrasmooth Patterned Metals for Plasmonics and Metamaterials. Science 2009, 325, 594-597.

9. Habteyes, T. G.; Dhuey, S.; Wood, E.; Gargas, D.; Cabrini, S.; Schuck, P. J.; Alivisatos, A. P.; Leone, S. R. Metallic Adhesion Layer Induced Plasmon Damping and Molecular Linker as a Nondamping Alternative. ACS Nano 2012, 6, 5702-5709.

10. Gallinet, B.; Siegfried, T.; Sigg, H.; Nordlander, P.; Martin, O. J. F. Plasmonic Radiance: Probing Structure at the Ångström Scale with Visible Light. Nano Lett. 2012, 13, 497-503.

11. Ye, J.; Wen, F.; Sobhani, H.; Lassiter, J. B.; Dorpe, P. V.; Nordlander, P.; Halas, N. J. Plasmonic Nanoclusters: Near Field Properties of the Fano Resonance Interrogated with SERS. Nano Lett. 2012, 12, 1660-1667.

12. Siegfried, T.; Kind, M.; Terfort, A.; Martin, O. J. F.; Zharnikov, M.; Ballav, N.; Sigg, H. Reusable Plasmonic Substrates Fabricated by Interference Lithography: a Platform for Systematic Sensing Studies. J. Raman Spectrosc. 2012, 44, 170-175.

13. Abdelsalam, M.; Bartlett, P. N.; Russell, A. E.; Baumberg, J. J.; Calvo, E. J.; Tognalli, N. G.; Fainstein, A. Quantitative Electrochemical SERS of Flavin at a Structured Silver Surface. Langmuir 2008, 24, 7018-7023.

14. Fromm, D. P.; Sundaramurthy, A.; Schuck, P. J.; Kino, G.; Moerner, W. E. Gap-Dependent Optical Coupling of Single Bowtie Nanoantennas Resonant in the Visible. Nano Lett. 2004, 4, 957-961.

15. Cui, B.; Clime, L.; Li, K.; Veres, B. Fabrication of Large Area Nanoprism Arrays and their Application for Surface Enhanced Raman Spectroscopy. Nanotechnology 2008, 19, 145302.

16. Aouani, H.; Wenger, J.; Gèrard, D.; Rigneault, H.; Devaux, E.; Ebbesen, T. W.; Mahdavi, F.; Xu, T.; Blair, S. Crucial Role of the Adhesion Layer on the Plasmonic Fluorescence Enhancement. ACS Nano 2009, 3, 2043-2048.

17. Barchiesi, D.; Macías, D.; Belmar-Letellier, L.; van Labeke, D.; Lamy de la Chapelle, M.; Toury, T.; Kremer, E.; Moreau, L.; Grosges, T. Plasmonics: Influence of the Intermediate (or Stick) Layer on the Efficiency of Sensors. Appl. Phys. B: Lasers Opt. 2008, 93, 177-181.

18. Najiminaini, M.; Vasefi, F.; Kaminska, B.; Carson, J. J. L. Optical Resonance Transmission Properties of Nano-Hole Arrays in a Gold Film: Effect of Adhesion Layer. Opt. Express 2011, 19, 26186-26197.

19. Sexton, B. A.; Feltis, B. N.; Davis, T. J. Characterisation of Gold Surface Plasmon Resonance Sensor Substrates. Sens. Actuators, A 2008, 141, 471-475.

20. Lahiri, B.; Dylewicz, R.; De La Rue, R. M.; Johnson, N. P. Impact of Titanium Adhesion Layers on the Response of Arrays of Metallic Split-Ring Resonators (SRRs). Opt. Express 2010, 18, 11202-11208.

21. Lamy de la Chapelle, M.; Shen, H.; Guillot, N.; Frémaux, B.; Guelorget, B.; Toury, T. New Gold Nanoparticles Adhesion Process Opening the Way of Improved and Highly Sensitive Plasmonics Technologies. Plasmonics 2012, 1-5.

22. Kern, A. M.; Meixner, A. J.; Martin, O. J. F. MoleculeDependent Plasmonic Enhancement of Fluorescence and Raman Scattering near Realistic Nanostructures. ACS Nano 2012, 6, 9828-9836.
23. Djaker, N.; Hostein, R.; Devaux, E.; Ebbesen, T. W.; Rigneault, $\mathrm{H}$.; Wenger, J. Surface Enhanced Raman Scattering on a Single Nanometric Aperture. J. Phys. Chem. C 2010, 114, 16250-16256.

24. Roxworthy, B. J.; Toussaint, K. C. Plasmonic Nanotweezers: Strong Influence of Adhesion Layer and Nanostructure Orientation on Trapping Performance. Opt. Express 2012, 20, 9591-9603.

25. Jiao, X.; Goeckeritz, J.; Blair, S. Oldham, M., Localization of Near-Field Resonances in Bowtie Antennae: Influence of Adhesion Layers. Plasmonics 2009, 4, 37-50.

26. Jeppesen, C.; Mortensen, N. A.; Kristensen, A. The Effect of Ti and ITO Adhesion Layers on Gold Split-ring Resonators. Appl. Phys. Lett. 2010, 97, 263103.

27. Goss, C. A.; Charych, D. H.; Majda, M. Application of (3-Mercaptopropyl)trimethoxysilane as a Molecular Adhesive in the Fabrication of Vapor-Deposited Gold Electrodes on Glass Substrates. Anal. Chem. 1991, 63, 85-88.

28. Päivänranta, B.; Langner, A.; Kirk, E.; David, C.; Ekinci, Y. Sub-10 nm Patterning using EUV Interference Lithography. Nanotechnology 2011, 22, 375302.

29. Siegfried, T.; Ekinci, Y.; Solak, H. H.; Martin, O. J. F.; Sigg, H. Fabrication of Sub-10 nm Gap Arrays over Large Areas for Plasmonic Sensors. Appl. Phys. Lett. 2011, 99, 263302.

30. Gallinet, B.; Kern, A. M.; Martin, O. J. F. Accurate and Versatile Modeling of Electromagnetic Scattering on Periodic Nanostructures with a Surface Integral Approach. J. Opt. Soc. Am. A 2010, 27, 2261-2271.

31. Gallinet, B.; Martin, O. J. F. Scattering on Plasmonic Nanostructures Arrays Modeled with a Surface Integral Formulation. Photonics Nanostruct. Fundam. Appl. 2010, 8, 278284.

32. Ebbesen, T. W.; Lezec, H. J.; Ghaemi, H. F.; Thio, T.; Wolff, P. A. Extraordinary Optical Transmission through Subwavelength Hole Arrays. Nature 1998, 391, 667-669.

33. Wu, S.; Wang, Q.-j.; Yin, X.-g.; Li, J.-q.; Zhu, D.; Liu, S.-q.; Zhu, Y.-y. Enhanced Optical Transmission: Role of the Localized Surface Plasmon. Appl. Phys. Lett. 2008, 93, 101113.

34. McFarland, A. D.; Young, M. A.; Dieringer, J. A.; Van Duyne, R. P. Wavelength-Scanned Surface-Enhanced Raman Excitation Spectroscopy. J. Phys. Chem. B 2005, 109, 1127911285.

35. Barnes, W. L.; Dereux, A.; Ebbesen, T. W. Surface Plasmon Subwavelength Optics. Nature 2003, 424, 824-830.

36. Otte, M. A.; Estavez, M. C.; Carrascosa, L. G.; GonzalezGuerrero, A. B.; Lechuga, L. M.; Sepalveda, B. Improved Biosensing Capability with Novel Suspended Nanodisks. J. Phys. Chem. C 2011, 115, 5344-5351.

37. Abelmann, L.; Lodder, C. Oblique Evaporation and Surface Diffusion. Thin Solid Films 1997, 305, 1-21.

38. Sopra, refraction indices database. www.refractiveindex. info.

39. Yang, C.-C.; Chen, W.-C. The Structures and Properties of Hydrogen Silsesquioxane (HSQ) Films Produced by Thermal Curing. J. Mater. Chem. 2002, 12, 1138-1141.

40. Johnson, P. B.; Christy, R. W. Optical Constants of the Noble Metals. Phys. Rev. B 1972, 6, 4370-4379. 